\title{
Development of Deep Learning Algorithm using Convolutional Neural Network for Medical Imaging
}

\author{
Kunal S Khadke
}

\begin{abstract}
Medical imaging is the procedure and approach of formulating graphic models of the peculiarity of a body system for medical investigation and treatment, and also graphical illustration of the function of several internal organs or structures. To identify the affected tissues of the brain in a case of brain tumors, it is important to get high precision and accuracy to locate exact pixels. Manual analysis may be erroneous and so it is important to use deep learning image segmentation technique. Segmentation of graphic is the technique of dividing a graphic in to several group of pixels. The earlier objective of the segmentation is actually to produce details much easier and enhance the manifestation of clinical images into significant content. Segmentation is a complicated activity due to the excessive variability in the graphics. The computational intelligence is modern way for application automation. Existing studies shows need of deep learning research for fast and accurate medical imaging solutions. Hence, this paper presents the CNN framework (for an analysis of brain tumors) as a base for further research methodology development. The paper also provides a pilot research analysis that can further be used to develop improved precision and visibility.
\end{abstract}

Keywords: deep learning, CNN, brain tumor, U-cnet, feature extraction, segmentation, augmentation, machine learning.

\section{INTRODUCTION}

Deep learning (DL) is a sub-domain of machine learning [1] as well as, lately proved impressive capabilities specifically in classification as well as, segmentation conditions. In this paper, a DL model influenced by a convolutional neural network is recommended to identify numerous brain tumor aspects that implement freely obtainable datasets. Brain tumors are actually the impact of disproportionate clumps and unchecked cells intensity the brain. They are able to result in loss of life if they happen to be far from diagnosed promptly and effectively. Several aspects of brain tumor including Meningioma, Glioma, and so Pituitary tumors are actually much more routine as opposed to the other types. Meningiomas are actually the very well-known class of tumors which usually develop in the narrow walls which usually enclose the brain as well as, vertebral cord. Meningiomas tumors are quite often non-cancerous. The Gliomas are actually a group of tumors which usually

Revised Manuscript Received on February 05, 2020.

* Correspondence Author

Kunal S Khadke, Assistant Professor, Department of Computer Engineering, PES Modern College of Engineering, Pune, India.

(C) The Authors. Published by Blue Eyes Intelligence Engineering and Sciences Publication (BEIESP). This is an open access article under the CC BY-NC-ND license (http://creativecommons.org/licenses/by-nc-nd/4.0/) develop through the element of the brain and simply sometimes incorporate with the ordinary brain tissues [2]. Gliomas tumors provoke a fairly little life span in cases where the overall size of the growth is reasonably significant. Pituitary cancer is an uncommon development of the brain structure. Pituitary cancer commonly improves, refining in the pituitary boucle within the brain. Several pituitary tumors result while in the uncommon and serious growth in the human hormones which usually reinstate necessary capabilities of the physique. This kind of cancer can glimpse any place right from the brain simply because of their whole innate aspect. Additionally, they will accomplish not likely feature a steady pattern. They can come with many general sizes, patterns, and contrasts. Magnetic Resonance Imaging (MRI) is a medical imaging approach, which generally is frequently accustomed for investigation and medication of brain tumors in surgical perform. The MR images are undertaken from 3 assorted directions. Many of these sights are termed sagittal, orthogonal and coronal.

Brain Tumor segmentation practices are a significant element in cancer recognition. Utilizing machine learning practices which usually discover the structure of brain tumor is very helpful due to normal segmentation is time consuming as well as, obtaining predisposed to individuals mistakes or glitches. In standard therapeutic graphic segmentation, it is the technique of automated or simply semi-automatic recognition of border inside a 2D or 3D graphic. Even though certainly, there are present different approaches for brain tumor distinction, however these have several boundaries which in turn require to be viewed as though functioning with brain tumor segmentation as well as category $[3,4]$. In present times various functions appear to have been implemented for segmentation of therapeutic images, just like epidermis lesion, brain tumor prognosis, monitoring heart ventricles, and so liver organ investigation. Generally, healthful brain cells consist of 3 units: dark subject, white colored subject, and cerebrospinal substance. The segmentation is employed to discover spaces enclosed by a tumor. The segmentation ought to isolate the established malignant cells from the necrotic cells, and as well, equally the edema needs to be discovered. This can be performed by figuring out unusual spaces in the event that reviewed to regular cells. Several automated brain tumor segmentation solutions employ hand crafted functionality just like sides, angles, histogram of incline, localized binary structure, etc [5,6,]. Generally, there may be a vital attempt in producing conventional machine learning algorithms for segmentation of typical and unusual brain cells in MRI. 


\section{Development of Deep Learning Algorithm using Convolutional Neural Network for Medical Imaging}

Also, transformation of the image helps such segmentation which needs cautious the anatomist and the particular competence [7]. Figure 1 shows the steps for feature extraction.

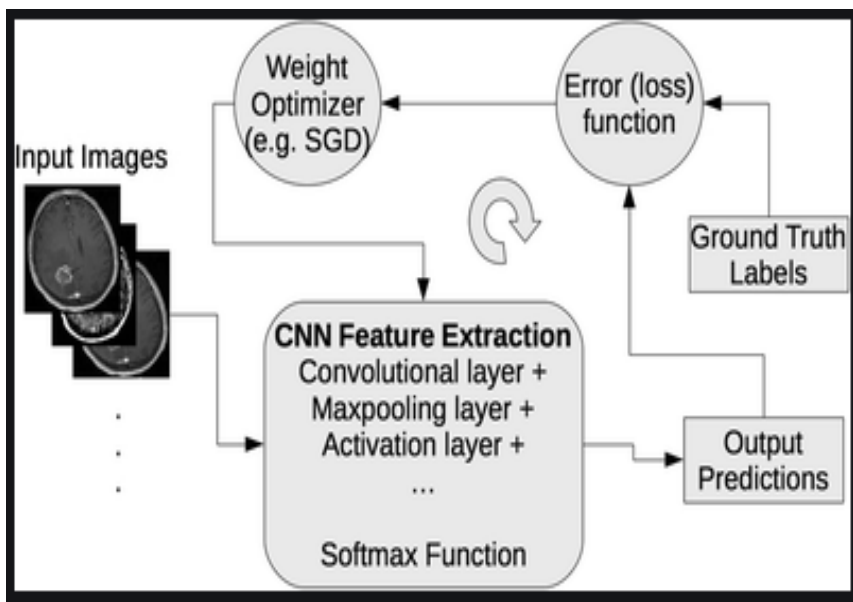

Fig. 1. A schematic representation of CNN training [7]

Through these kinds of strategies, the emphasis is an on the execution of a conventional machine learning pipeline. The supposed abilities are initially taken out and so provided to a classifier. The training process of the classifier is not really influenced by the element of these aspects. Convolutional neural networks (CNNs) will not employ hand crafted aspects and so these features were utilized productively to segmentation ailments.

The brain is the control facility of our central nervous structure and so is accountable meant for the performance of actions most through the individual body system. Brain tumors can certainly impact individual lifestyles immediately. In case the tumor is determined within a premature level, the patient's endurance likelihood advances. Prognosis of a brain tumor is a complicated challenge because of the excessive range in tumor presence and suspect tumor borders. MRI pictures are selected for the diagnosis of brain tumors, as these will be utilized in the conviction of soft cells. Initially, graphic pre-processing is utilized to strengthen graphic standard [8] images can be frequently applied by doctors to be able to identify the presence of tumors or simply the options of the tumors. The degree of brain tumor medication depends on the physician's knowledge and experience. Because of this, by using an automatic and faultless performing tumor prognosis strategy is tremendously essential to help doctors to sense brain tumors. Recognition of tumors in the brain by means of MR graphics features grown into a vital activity and so various research have already been executed in the latest years.

In deep learning for clinical image resolution technology, a computational design discovers the classification work immediately from graphics. Deep learning designs can attain cutting-edge correctness, occasionally beyond human-level capabilities [9, 10]. Models are get trained by utilizing a significant set of marked records and neural network architectures that possess numerous levels. The proposed research will be conducted to perform the tumor segmentation and survival period calculation with development of new deep learning algorithm. The proposed research can be very useful for medical domain. Unlike simple MRI image where only feature extraction can be done,

deep learning is a backbone of artificial intelligence which is latest technology to develop a more accurate image processing tool.

\section{PROPOSED METHODOLOGY}

Electronic graphics engage in an important task on a day-to-day basis. The healthcare image application alludes to managing graphics by employing the computer. This particular application incorporates numerous forms of strategies and functions just like graphic gaining, safe-keeping, appearance, and interaction [11]. The image is a functionality that usually denotes an estimate of elements just like visibility or coloration a considered vision. The electronic images possess various advantages including speedier and affordable refinement expense, uncomplicated storage and transmission, instantaneous quality analysis, an array of copying with saving the quality, rapidly as well as economical processing, and handy modification [12]. The drawbacks of electronic graphics are production re-tighten, incapacity to resize with retaining the quality, the desire of large-capacity storage, and the requirement of more rapid processer for modification.

In a computer system prospects, CNN-based segmentation designs have got usually employed to genuine scenario marking. For all these steps, the advises for the version will be the RGB options of a plot via a color graphic. Present analysis functions a primary CNN to produce estimations for each and every pixel and also additionally elevates the estimations by employing them all as excess data in the source of a secondly CNN design. Much other work includes a number of distinctive CNNs controls of the graphic at numerous resolutions. To generate a consistent segmentation, these kinds of estimations will need to get regularized by using an additional universal excellent pixel segmentation of the graphic [13]. A popular issue in actual living programs of deep learning founded classifiers is which certain courses have got an appreciably greater variety of cases in the training collection rather than several other instructions. This particular variance is related to group asymmetry. Generally, there are an abundance of samples in fields like computer vision [14], clinical prognosis [15], and fraudulence discovery [16] and somewhere by this approach challenge is tremendously important and the occurrence of an individual group might be 1000 times fewer as opposed to another group. Because of organized nature that may group difference are able to have sizeable undesirable consequence on workout conventional classifiers which include multi-layer perceptions [17]. It influences either concurrence through the training step and generalization of a shape on the assessment set. Furthermore, Radiomics represents a wide-ranging collection of computational procedures that acquire quantitative attributes through radiographic graphics. The ensuing attributes are often implemented to notify visual prognosis, diagnosis, and therapies solution in oncology. Furthermore, Radiomics represents a wide-ranging collection of computational procedures that acquire quantitative attributes through radiographic graphics. 
The ensuing attributes are often implemented to notify visual prognosis, diagnosis, and therapies solution in oncology.

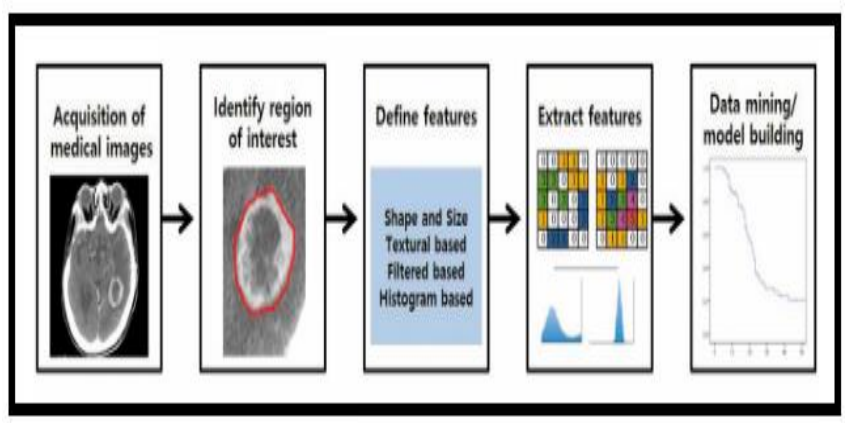

Fig. 2. Block diagram of Traditional Radiomics model (Source: IJCRT)

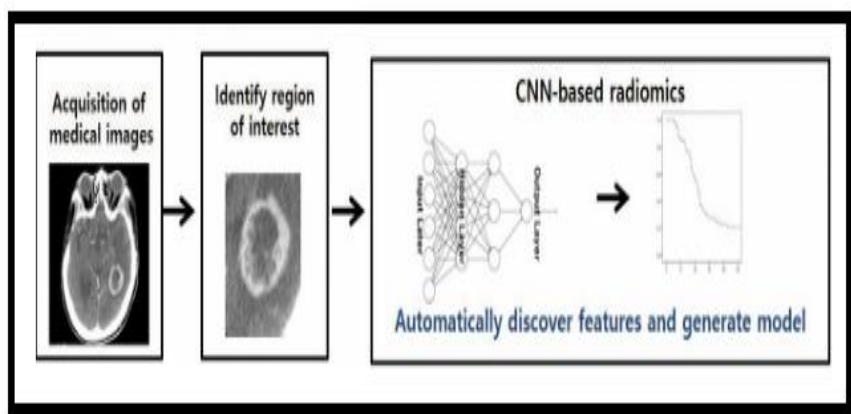

Fig. 3. Block diagram of CNN based Radiomics model (Source: IJCRT)

Nevertheless, important problems continue to be for methodological improvements to enhance feature extraction and present immediate details rate in health-related controls. Similarly, significant, to be scientifically important, predictive radiomic attributes have to be certainly associated to significant biologic properties and qualitative solution components familiarized to radiologists. In this article author employed a cross-disciplinary procedure to showcase analyses in radiomics [18]. Radiomics evaluation for the computerized diagnosis in neural growth individuals employs an extensive variety of visualize capabilities calculated through the region of interest (ROI) as the source to a classifier unit. Conventional radiomics strategies depend on a number of hand-crafted attributes, for illustration, dependent on histograms of high intensity, grey levels co-occurrence matrix, location gray-tone variance matrix as well as gray-level region dimension matrix. In the last decade, CNNs has obtained state-of-art functionality for a huge variety of graphic classification duties. A CNN is a multi-layered design that offers spatial framework and weight posting among pixels or voxels. As opposed to typical radiomic strategies, which often depend on hand-crafted attributes to encode graphics, CNNs primarily understand graphic illustrations which are effortless for category steps, specifically from training records [19]. In clinical imaging, the accessibility of records is particularly restricted for developing a deep CNN. Furthermore, fewer records may result in an overfit CNN. This difficulty could be considerably mitigated by applying graphic enhancement to build considerably more training details. Otherwise, transfer learning can certainly be applied, which inturn is a procedure of implementing recently discovered information in a latest area[20]. The primary building block applied to develop a CNN structure is the convolutional layer. A number of layers can be piled on the finest of every different developing a structure of capabilities. Each layer is often realized as taking out features from its earlier layer within the structure to which it is linked.

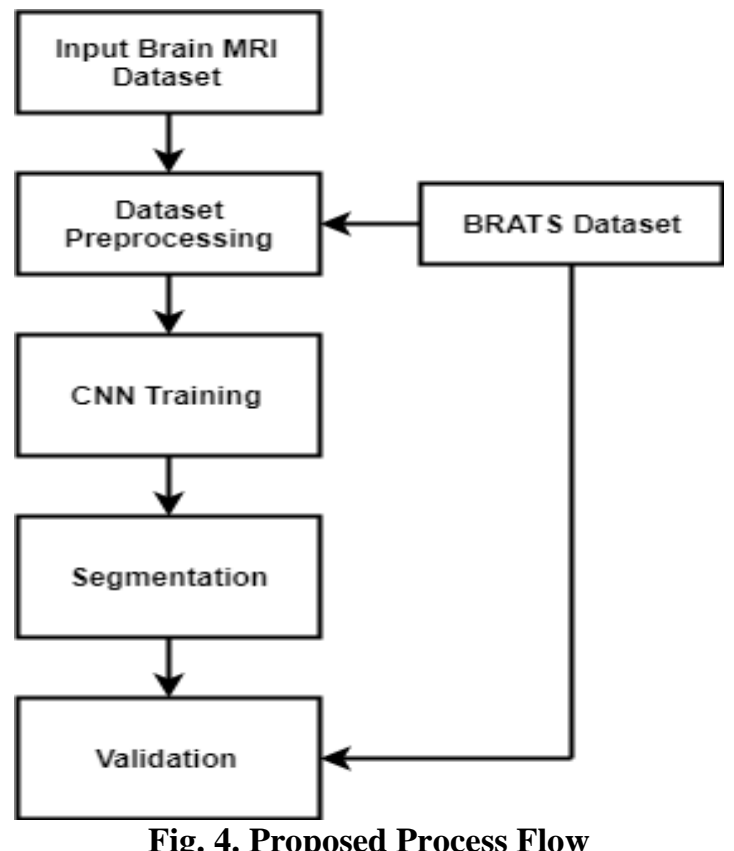

An individual convolutional layer will take as source a bunch of input planes and generate as the end result some variety of end result planes or characteristic maps. Each and every characteristic map might be considered as a topologically organized map of responses of a unique spatially neighborhood non-linear characteristic extractor, employed in the same way to each one space community of the source planes in a sliding window manner.

\section{Proposed Algorithm: K-means-Extraction}

1. Input Brain MRI Dataset DBArr[i] \# input image for feature extraction

2.\# Read the image from dataset, splitting by pixels as gray and white pixels

3. $\mathrm{P}$ = open(imageName, ' $r$ '); \# open image file

4. pixelGray = P.read().readGray(); \# identify gray pixel

5. pixelWhite $=$ P.read() readWhite(); \# identify white pixel

6. DBArr= [i]; \# read image ' $i$ ' as a set of gray pixels

7. for $\mathrm{i}$ in range $(0$, pixelGray $(\mathrm{P}))$ : \# read array of gray pixels

8. extractFeatures(); \# feature extraction function

9. for $\mathrm{j}$ in range(pixelGray $(\mathrm{P})-1)$ :

10. extractFeatures.append(i);

11. imageName.append(extractFeatures); \# lable image for identified features

12. return imageName;\# processed image will be returned As shown in algorithm above, the k-means cluster formation further used to identify gray pixels (i.e. affected tumor) and white pixel cluster (i.e. normal tumor tissues). These features will be used with CNN. In the circumstance of an initial convolutional layer, the specific source planes match distinct MRI types. In succeeding tiers, the sources planes are commonly consisting of the characteristic maps of the preceding layer.

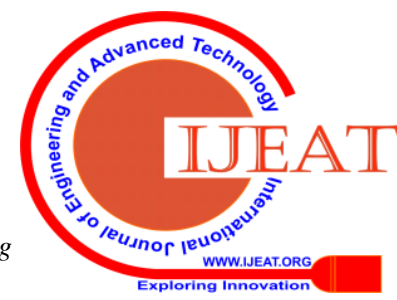




\section{Development of Deep Learning Algorithm using Convolutional Neural Network for Medical Imaging}

\section{RESULTS AND ANALYSIS}

The proposed algorithm with CNN as a base framework gives following accuracy for BRATS 2018 dataset of T1, T2 stage tumors.

Table-I: Output Features of BRATS 2018 dataset

\begin{tabular}{|c|c|c|}
\hline PatientID & $\begin{array}{c}\text { Manual } \\
\text { Accuracy for } \\
\text { MR image (\%) }\end{array}$ & $\begin{array}{c}\text { K-means } \\
\text { Cluster } \\
\text { Accuracy for } \\
\text { MR image (\%) }\end{array}$ \\
\hline Brats-2018-pid-65 & 12 & 14.5 \\
\hline Brats-2018-pid-65 & 16 & 18.5 \\
\hline Brats-2018-pid-65 & 18 & 20.7 \\
\hline
\end{tabular}

The U-cnet framework is used for proposed CNN extension development, which shows that the k-means cluster algorithm can improve accuracy. The manual analysis for brain MR images can be erroneous, and hence computational assistance is necessary for accurate reading about affected tumors. The pilot research shows that it can further be used for segmentation of brain tumors.

\section{CONCLUSION}

The proposed methodology can be very useful for brain MR image segmentation effectively. In this paper, we discussed various existing methods for deep learning practices. The $\mathrm{CNN}$ is used as a base framework along with U-cnet approach where pooling layers used to improve accuracy using k-means clustering algorithm for gray and white pixel cluster formation. The max-pooling provides the feature extraction to be included as a next level of analysis. Hence, deep learning objective is satisfied by reading each pixel and sub-pixels. The proposed research can be used as a base for robotics guided surgery by means of microprocessor programming. The future development can be done for BRATS 2018 dataset by using segmentation for tumor stages as T1, T2, T1ce etc. The survival rate of patients can be identified with the implementation of deep learning.

\section{REFERENCES}

1. Zhang, Chong, et al. "Brain Tumor Segmentation Based on Hybrid Clustering and Morphological Operations." International Journal of Biomedical Imaging 2019 (2019).

2. Tong, Jijun, et al. "MRI brain tumor segmentation based on texture features and kernel sparse coding." Biomedical Signal Processing and Control 47 (2019): 387-392.

3. Sajjad, Muhammad, et al. "Multi-grade brain tumor classification using deep CNN with extensive data augmentation." Journal of computational science 30 (2019): 174-182.

4. Afshar, Parnian, Konstantinos N. Plataniotis, and Arash Mohammadi. "Capsule Networks for Brain Tumor Classification Based on Mri Images and Coarse Tumor Boundaries." ICASSP 2019-2019 IEEE International Conference on Acoustics, Speech and Signal Processing (ICASSP). IEEE, 2019.

5. Gumaei, Abdu, et al. "A Hybrid Feature Extraction Method With Regularized Extreme Learning Machine for Brain Tumor Classification." IEEE Access 7 (2019): 36266-36273.

6. Hou, Le, et al. "Patch-based convolutional neural network for whole slide tissue image classification." Proceedings of the IEEE Conference on Computer Vision and Pattern Recognition. 2016.

7. Akkus, Zeynettin, et al. "Deep learning for brain MRI segmentation: state of the art and future directions." Journal of digital imaging 30.4 (2017): 449-459.

8. Miranda, Rodrigo Gondim. "Computational Intelligence-Based System in the Support of the Diagnosis of Brain Tumors: An Approach through Fuzzy C-Means Method." Journal of Pharmacy and Pharmacology 6 (2018): 626-628.

9. Schmidhuber, Jürgen. "Deep learning in neural networks: An overview." Neural networks 61 (2015): 85-117.

10. Cha, Young-Jin, Wooram Choi, and Oral Büyüköztürk. "Deep learning-based crack damage detection using convolutional neural networks." Computer-Aided Civil and Infrastructure Engineering 32.5 (2017): 361-378.

11. Pham, Chi-Hieu, et al. "Brain MRI super-resolution using deep 3D convolutional networks." 2017 IEEE 14th International Symposium on Biomedical Imaging (ISBI 2017). IEEE, 2017.

12. Li, Wenqi, et al. "On the compactness, efficiency, and representation of 3D convolutional networks: brain parcellation as a pretext task." International Conference on Information Processing in Medical Imaging. Springer, Cham, 2017.

13. Havaei, Mohammad, et al. "Brain tumor segmentation with deep neural networks." Medical image analysis 35 (2017): 18-31.

14. Buda, Mateusz, Atsuto Maki, and Maciej A. Mazurowski. "A systematic study of the class imbalance problem in convolutional neural networks." Neural Networks 106 (2018): 249-259.

15. Kaur, Bhavneet, et al. "An improved salient object detection algorithm combining background and foreground connectivity for brain image analysis." Computers \& Electrical Engineering 71 (2018): 692-703.

16.Zhang, Zhaohui, et al. "A model based on convolutional neural network for online transaction fraud detection." Security and Communication Networks 2018 (2018).

17. Díaz-Álvarez, Alberto, et al. "Modelling the human lane-change execution behaviour through Multilayer Perceptrons and Convolutional Neural Networks." Transportation research part F: traffic psychology and behaviour 56 (2018): 134-148.

18. Zhou, Mu, et al. "Radiomics in brain tumor: image assessment, quantitative feature descriptors, and machine-learning approaches." American Journal of Neuroradiology 39.2 (2018) 208-216.

19. Chaddad, Ahmad, et al. "Deep radiomic analysis based on modeling information flow in convolutional neural networks." IEEE Access 7 (2019): 97242-97252.

20. Paul, Rahul, et al. "Predicting Nodule Malignancy using a CNN Ensemble Approach." 2018 International Joint Conference on Neura Networks (IJCNN). IEEE, 2018.

\section{AUTHORS PROFILE}

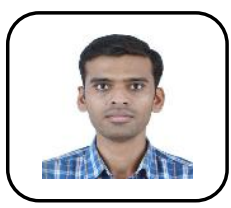

Kunal S Khadke, Assistant Professor, Department of Computer Engineering, Pune, India. 